\title{
Segmentation of Gated SPECT Images for Automatic Computation of Myocardial Volume and Ejection Fraction
}

\author{
P. Brigger, S. Bacharach, A. Aldroubi and M. Unser \\ Biomedical Engineering and Instrumentation Program, National Institutes of Health \\ Bethesda, MD 20892, USA
}

\begin{abstract}
We describe an image processing system for the automatic assessment of ejection fraction (EF) from noisy SPECT left ventricular myocardial perfusion images. A segmentation scheme consisting of an elliptical coordinate transformation, matched filtering and dynamic contour tracking detects the endo- and epicardial boundaries. Computation of EF is performed based on the epi-rather than the endo-cardial boundary, which proves to be more robust for images with low signal-to-noise ratios. The computation incorporates anatomical constraints of constant myocardial mass and smooth cardiac variation. The algorithm was tested on different image modalities and shows good linear agreement with EFs obtained from conventional approaches based on planar gated blood pool imaging (PET: $y=8.7+1.07 x, \quad r=0.84 \quad$, Technetium-99m MIBI SPECT: $y=5.0+0.80 x, r=0.90$, Thallium-201 SPECT: $y=23.5+0.82 x, \quad r=0.77, \quad R M S$ error $=10.0)$. The proposed scheme may be an alternative for EF computation without the need for additional image acquisitions.
\end{abstract}

\section{Introduction}

Diseases of the heart and its blood vessels result in a large morbidity and mortality in Western civilizations. Gated perfusion SPECT (Single Photon Emission Tomography) is a recent and promising new cardiac imaging technique allowing simultaneous measurement of both perfusion and cardiac function. Temporal information is obtained through gating, i.e. synchronization of image acquisition instances with the ECG. SPECT is relatively cheap compared to PET or MRI, and already used in over 5 million studies in the US each year. The images are obtained by injection of a radioactive tracer into the blood which perfuses into the myocardial heart muscle from where photons are emitted and measured using a Gamma camera. Homogeneously high uptake of the tracer signifies healthy tissue, whereas regions of low or no up-take imply ischemic or dead tissue. Physicians assess heart viability and perfusion by examination of uptake (often visually), i.e. a bright or dim image. However, gated perfusion SPECT could allow one to compute several other clinically important parameters, such as absolute heart volume, ejection fraction (EF) (i.e. a measure of the heart's efficiency), myocardial mass, and temporal evolution of wall thickening. EF or wall thickening is currently mainly obtained through separate, additional studies (e.g. gated blood pool imaging, echo, or MRI imaging). Only recently have algorithms been developed to compute EF directly [7, 6, 9]. Hence, the ability to quantify both perfusion and the additional functional information of EF from a single study could result in a reduction in cost, time and radiation dose.

In this paper, we propose a general technique for a robust and reliable boundary segmentation without the use of thresholds, as are often required $[6,9,11]$. We present a consistent approach of dealing with noisy image data (Section 2), as is typically the case with Thallium-201 SPECT images. The proposed technique is based on an elliptical coordinate transformation of the original data which naturally leads to a more robust segmentation using dynamic programming. Then, by incorporation of physiological constraints, EF is computed based on the epi-cardial boundary. In Section 3, validation methods and results are presented.

\section{Image processing system}

In this section, the segmentation block and the EFcomputation block depicted in Fig. 1 will be presented. We denote by $f_{t}(k, l)$ the original image and by $s_{t}(k, l)$ the segmented image, where the subscript denotes the gating instance.

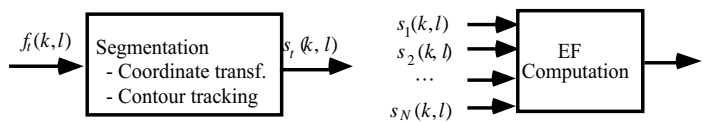

Figure 1: Block diagram of the image processing system 


\subsection{Elliptical coordinate transformation through multi-resolution Hough analysis}

The purpose of a change of coordinate system is twofold: 1) present the data in a suitable form for segmentation, 2) provide a system where true count profiles of the myocardium, perpendicular to the heart wall, can be measured. The apparent shape of the myocardium asks for an elliptical coordinate transformation (Fig. 2). The geometrical transformation maps the original image $f_{t}(k, l), k, l \in Z$ into an area of interest $g_{t}(i, j), \quad i, j \in Z$ in the transformed coordinate system, where $i$ relates to the radial angle and where $j$ is an index proportional to the distance from the ellipse center along a radial path $\mathbf{r}(p, q)$. The mathematical formulation of the coordinate transformation is explained in detail in [3].

Because of its ability to detect complex pattern, we have chosen the Hough transform (HT) [5] for the detection of the ellipse. The main computational difficulty is the huge size of the Hough parameter space [12]. We achieve high algorithm performance (precision, confidence) by using the gray-level values directly to weight HT votes instead of computing a binary image, as it is usually done [10]. The heighest votes will occur at the radial maxima indifferently of the relative height of these maxima. We achieve high computation performance through the use of a multiresolution analysis. A centered pyramid implementation [4] provides us with a common axis of symmetry at all pyramid levels. It allows an iterative bottom-up computation and a straightforward parameter up-projection. Without a multiresolution approach, computation time is 2.8 hours for a $128 \times 128$ image on a PowerPC. This time compares to $98 \mathrm{sec}$. when two resolution levels are used and to $53 \mathrm{sec}$. when three resolution levels are used.

\subsection{Constrained contour tracking by dynamic programming.}

Segmentation and wall thickness measurements of perfusion SPECT images have almost exclusively been based on one of the following three techniques: 1) Counts based segmentation, relating maximal counts in a radial count profile to the wall thickness. 2) Geometry based segmentation, performing a local threshold at half maximum of the count profile. Both techniques are highly non-linear for the heart's dimensions [8]. 3) Global thresholding gives only incomplete and very inaccurate boundaries because of non-homogeneous count profiles. All three techniques require images with relatively good up-takes, which is often not given in practice.

For our application, we must design an algorithm which is robust in noisy environments, and detects a single, smooth and connected contour (endo- and epicardial boundaries are considered as two separate problems). The problem can be reformulated as an optimization problem. We search for a contour segment $T=\left[\left(1, j_{1}\right),\left(2, j_{2}\right), \ldots,\left(i, j_{i}\right), \ldots,\left(N, j_{N}\right)\right], \quad$ satisfying the constraints and maximizing some criterion $\varsigma\left(T_{k}\right)$. We use the same maximization criterion as in [13], i.e. the cumulative sum of vertical derivatives about the contour segment:

$$
\varsigma\left(T_{k}\right)=\sum_{i=1}^{N}\left[f\left(i, j_{i}-1\right)-f\left(i, j_{i}+1\right)\right]
$$

With these definitions, the approach by dynamic programming [2] allows us to find the optimal contour by searching for sub-trajectories that fulfill (1). The incremental algorithm then becomes extremely efficient and the correct contour is computed on a low end workstation (PowerPC, $120 \mathrm{MHz}$ ) in a fraction of a second. The various image processing steps are illustrated in Fig. 2 (using less noisy data, to better illustrate each step) and two segmentation results are given in Fig. 3.

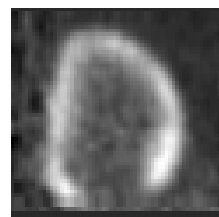

a) Original

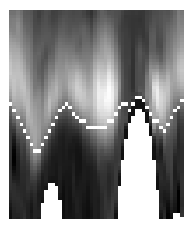

c) Contour

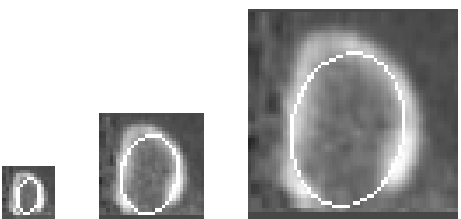

b) Multi-resolution decomposition

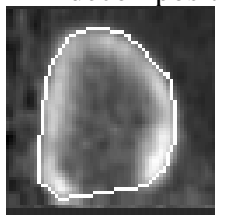

d) Segmentation tracking
Figure 2: Demonstration of the various image processing steps

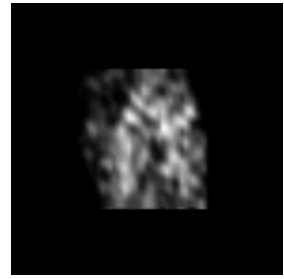

a)

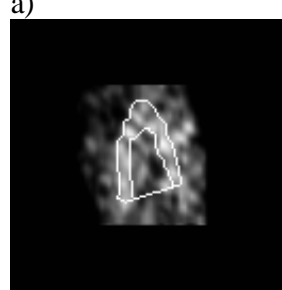

c)

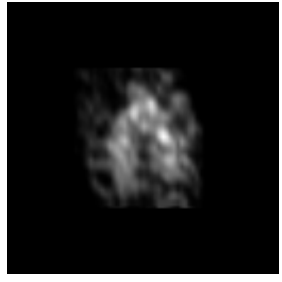

b)

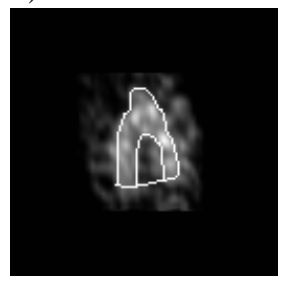

d)
Figure 3: Segmentation results. a) and b) : Original TlSPECT images. c) and d) Segmentation of $a$ ) and b) 


\subsection{EF computation based on the epi-cardial contours}

For each time point $t$, the segmentation block provides us with absolute values of the volume of the endo-cardium, $V_{\text {endo }}(t)$, and the epi-cardium, $V_{e p i}(t)$. Ejection fraction can now be computed as

$$
E F=\frac{V_{\text {endo }}\left(t_{D}\right)-V_{\text {endo }}\left(t_{S}\right)}{V_{\text {endo }}\left(t_{D}\right)}
$$

where $V_{\text {endo }}\left(t_{D}\right)=\max _{t}\left[V_{\text {endo }}(t)\right]$ is the end diastolic volume and $V_{\text {endo }}\left(t_{S}\right)=\min _{t}\left[V_{\text {endo }}(t)\right]$ is the end-systolic volume.

In the literature, (2) has been used exclusively to compute EF. However, it is not optimal for two reasons. 1) Equation (2) is extremely sensitive to noise because the result is based on only two values out of the $N_{t}$ gating instances (the maximum and minimum value, respectively). 2) The endo-cardial boundary is less accurate than the epi-cardial boundary. This is because perfusion profiles of opposite walls may add up when the walls are close, which is the case especially at systole.

We can reduce the effect of the noisy data (which is particularly apparent for Tl-SPECT images) by considering all gating instances. It has been found in [1] that the volume curve can be described by two harmonics of the Fourier description. Hence, we smooth the data by computing its Fourier transformation, retaining the two most significant harmonics, and reconstructing the data. Systole and diastole gating times are then found as the minimum and maximum of the smoothed data.

Addressing the second point mentioned before, we propose to use the epi-cardial boundary to compute EF. It is less affected by profile addition and therefore can be segmented more reliably. The endo-cardial volume can be re-written as $V_{\text {endo }}(t)=V_{\text {epi }}(t)-m$, where $m$ is the volume of the myocardium, and therefore $\mathrm{EF}$ is

$$
E F=\frac{V_{e p i}\left(t_{D}\right)-V_{e p i}\left(t_{S}\right)}{V_{e p i}\left(t_{D}\right)-m}
$$

The computation of the myocardial mass, $m$, is obtained through averaging of the difference between epicardial and endo-cardial volume over the entire gating cycle. Therefore, the effect of outliers in the endo-cardial volume is reduced and the precision increased by a factor of $\sqrt{N_{t}}$.

This approach has not been considered before. We will show experimentally that it produces the same results for noiseless images and better results for noisy images.

\section{Results}

The algorithms were tested on images obtained from three different modalities. 1) Gated FDG-PET using 5m $\mathrm{Ci}$ of ${ }^{18} \mathrm{~F}$-fluorodeoxyglucose, 17 patients, 2) Gated ${ }^{99 \mathrm{~m}} \mathrm{Tc}$ - sestamibi SPECT, 9 patients, 3) Gated Thallium-201 SPECT, 32 subjects. In addition, we constructed a simulated Tl-SPECT image sequence by adding real SPECT noise to the FDG-PET images to obtain similar image statistics (SNR ratios). The simulated Tl-SPECT sequence will be called PET-SPECT.

In a first experiment, we evaluated the effectiveness of computing EF based on the epi-cardial contour (3) vs. a computation based on the endo-cardial contour (2). For this purpose, we compared both types of measurements for the noise free PET images and the noisy PET-SPECT images. In the former case, a relatively good correlation should be observed, whereas in the latter case more fluctuations and a less good linear agreement can be expected. It was then interesting to compare $\mathrm{EF}$ computation for the PET images vs. a computation for the PET-SPECT images based on the epi-cardial contour and the endo-cardial contour, respectively. The better the respective data correlates, the more EF computation is stable. The respective results are shown in Table I. A very good linear agreement $(\mathrm{r}=0.92)$ can be observed between the two types of measurements for the PET image. A calibration of the derivation operator in (1) could be applied to obtain unity slope. Hence, for noiseless images, both (2) and (3) give essentially the same result. For the PET-SPECT images, the linear agreement is less good, and one needs to determine which formulation of $\mathrm{EF}$ is more accurate. The answer is given in rows three and four of Table I. Here, EFs obtained from the noiseless PET images and obtained from the noisy PET-SPECT images are compared using the same formula. Ideally, a unity correlation coefficient should be obtained. Clearly, computation of EF based on the epi-cardial contour (3) is more accurate for noisy images. For noiseless images, either formula (2) or (3) may be used.

A second experiment shows the relationship between the automatically computed EF measurements on gated PET, Tc-SPECT and Tl-SPECT images and EFs obtained from gated planar blood pool imaging. All the sets show a good linear agreement

(PET: $y=8.7+1.07 x, r=0.84$,

Tc-SPECT: $\quad y=5.0+0.80 x, r=0.90, \quad$ Tl-SPECT: $y=23.5+0.82 x, r=0.77$, RMS error $=10.0$ ). Some of the segmented as well as the original image sequences can be dynamically viewed over the World Wide Web (http://picasso.ncrr.nih.gov/brigger/SPECT). The quicktime movies allow a direct visualization of the algorithm's ability to interpolate over perfusion defects and how it can handle images of low signal-to-noise ratio. 


\section{Table IV}

Comparison of the formulas for EF based on the endo-cardial contours $\left(E F_{E N D O}=\frac{V_{\text {endo }}\left(t_{D}\right)-V_{\text {endo }}\left(t_{S}\right)}{V_{\text {endo }}\left(t_{D}\right)}\right)$ and on the epi-cardial contours $\left(E F_{E P I}=\frac{V_{e p i}\left(t_{D}\right)-V_{e p i}\left(t_{S}\right)}{V_{e p i}\left(t_{D}\right)-m}\right)$. Compared are EFs for the noise free PET image and for the noisy PET-SPECT image.

\begin{tabular}{ll|l} 
& & EF correlation \\
\hline PET: & $E F_{E N D O}$ vs. $E F_{E P I}$ & $y=7.2+1.50 \cdot x, \quad r=0.92$ \\
PET-SPECT: & $E F_{E N D O}$ vs. $E F_{E P I}$ & $y=5.9+1.46 \cdot x, \quad r=0.72$ \\
\hline$E F_{E N D O}:$ & PET vs. PET-SPECT & $y=13.3+0.54 \cdot x, \quad r=0.75$ \\
$E F_{E P I}:$ & PET vs. PET-SPECT & $y=8.3+0.80 \cdot x, \quad r=0.86$
\end{tabular}

\section{Conclusions}

We have presented a general segmentation algorithm for the detection of endo- and epi-cardial boundaries in noisy and noiseless nuclear cardiac image sequences (PET, Tc-SPECT, and Tl-SPECT). The algorithms operate with a very limited number of threshold and tuning parameters. Indeed, for all image modalities, the same input parameters were used and yielded good results. The feature makes the algorithms user-friendly and generally applicable. We have also introduced a novel approach to compute EF based on the epi-cardial boundary. Experimentally, it was shown that such an approach is more reliable when the original image data are noisy. Comparison of automatically computed values of EF to those obtained from gated blood pool imaging shows good linear agreement. We believe that these findings can give new insight in left ventricular function and that our methods can be used to improve many of the existing algorithms.

\section{References}

[1] S.L. Bacharach, M.V. Green, D. Vitale, G. White, M.A. Douglas, R.O. Bonow and S.M. Larson, "Optimum Fourier Filtering of Cardiac Data: A Minimum-Error Method: Concise Communication", Journal of Nuclear Medicine, vol. 24, no. 12, pp. 1176-1184, December, 1983.

[2] R. Bellman, Dynamic Programming. Princeton, NJ: Princeton University Press, 1957.

[3] P. Brigger, S.L. Bacharach, G. Srinivasan, K.A. Nour, V. Dilsizian, A. Aldroubi and M. Unser, "Segmentation of gated Tl-201-SPECT images for automatic computation of myocardial volume and ejection fraction", Journal of Nuclear Medicine, 1997. Submitted for publication.

[4] P. Brigger, F. Mueller, K. Illgner and M. Unser, "Centered pyramids", IEEE Transactions on Image Processing, 1996. Submitted for publication.

[5] R.O. Duda and P.E. Hart, "Use of the Hough Transformation To Detect Lines and Curves", Communications of the ACM, vol. 15, pp. 11-15, 1972.
[6] G. Germano et. al., "Automatic quantification of ejection fraction from gated myocardial perfusion SPECT", The Journal of Nuclear Medicine, vol. 36, no. 11, pp. 21382147, November, 1995.

[7] G. Germano, J. Erel, H. Kiat, P. Kavanagh and D. Berman, "Quantitative LVEF and qualitative regional function from gated Thallium-201 perfusion SPECT", Jour. Nuc. Medicine, vol. 38, pp. 749-754, 1987.

[8] E.J. Hoffman, S.C. Huang and M.E. Phelps, "Quantitation in Positron Emission Computed Tomography: 1. Effect of object size", Journal of Computer Assisted Tomography, vol. 3, no. 3, pp. 299308, June, 1979.

[9] K. Nichols, E.G.D. Puey and A. Rozanski, "Automation of gated tomographic left ventricular ejection fraction", Journal of Nuclear Cardiology, vol. 3, no. 6, pp. 475482, November/December, 1996.

[10] F. O'Gorman and M. Clowes, "Finding picture edges through collinearity of feaure points", in 3rd Int. Joint Conf. Artificial Intelligence, pp. 543-555, Stanford, August, 1973.

[11] G. Porenta and e. al., "Parameter Estimation of cardiac geometry by ECG-Gated PET imaging: validation using magnetic resonance imaging and echocardiography", Journ. of Nuclear Medicine, vol. 36, pp. 1123-1129, 1995.

[12] S. Tsuji and F. Matsumoto, "Detection of ellipses by modified Hough transformation", IEEE Transcations on Computers, vol. 27, pp. 777-781, 1978.

[13] M. Unser and M. Stone, "Automated detection of the tongue surface in sequences of ultrasound images", $J$. Accoust. Soc. Am., vol. 91, no. 5, pp. 3001-3007, May, 1992. 